\title{
Integrable impurity spin ladder systems
}

\author{
Arlei Prestes Tonel ${ }^{1}$, Angela Foerster ${ }^{1,2}$, Xi-Wen Guan ${ }^{1}$ and Jon Links ${ }^{1,3}$ \\ ${ }^{1}$ Instituto de Física da UFRGS, Av. Bento Gonçalves 9500, Porto Alegre, RS, Brazil \\ 2 Institut für Theoretische Physik, Freie Universität at Berlin, Arnimallee 14, 14195 Berlin, \\ Germany \\ ${ }^{3}$ Department of Mathematics, The University of Queensland, Queensland, 4072, Australia \\ E-mail: prestes@if.ufrgs.br, angela@if.ufrgs.br, guan@if.ufrgs.br and jrl@maths.uq.edu.au
}

Received 11 September 2002, in final form 8 November 2002

Published 17 December 2002

Online at stacks.iop.org/JPhysA/36/359

\begin{abstract}
Two different types of integrable impurities in a spin ladder system are proposed. The impurities are introduced in such a way that the integrability of the models is not violated. The models are solved exactly with the Bethe ansatz equations as well as the energy eigenvalues obtained. We show for both models that a phase transition between gapped and gapless spin excitations occurs at a critical value of the rung coupling $J$. In addition, the dependence of the impurities on this phase transition is determined explicitly. In one of the models the spin gap decreases by increasing the impurity strength $\Lambda$. Moreover, for a fixed $\Lambda$, a reduction in the spin gap by increasing the impurity concentration is also observed.
\end{abstract}

PACS numbers: 75.10.Jm, 71.10.Fd, 03.65.Fd

\section{Introduction}

The study of spin ladder systems continues to generate activity in order to understand the crossover from one dimension to two dimensions in condensed matter physics. In fact, with the rapid progress presently being made in nano-engineering, several compounds have been experimentally realized with a ladder structure, such as $\mathrm{La}_{1-x} \mathrm{Sr}_{x} \mathrm{CuO}_{2.5}$, $\mathrm{Sr}_{14-x} \mathrm{Ca}_{x} \mathrm{Cu}_{24} \mathrm{O}_{41}, \mathrm{Cu}_{2}\left(\mathrm{C}_{5} \mathrm{H}_{12} \mathrm{~N}_{2}\right)_{2} \mathrm{Cl}_{4}, \mathrm{CaV}_{2} \mathrm{O}_{5}$ (see, for example, [1, 2]). In addition, experiments using techniques such as magnetic susceptibility measurements [3] or nuclear magnetic resonance [4] report the existence of a spin gap in the spectrum of elementary excitations for even leg ladders. The existence of such a spin gap is crucial for superconductivity to occur under hole doping, as verified experimentally in some of these compounds and also predicted theoretically. In this context, effects of impurities in ladder systems have also been investigated. Experiments of angle resolved photoemission and Raman spectroscopy carried out in different ladder compounds, such as $\mathrm{Bi}_{2} \mathrm{Sr}_{2} \mathrm{CaCu}_{2} \mathrm{O}_{8}, \mathrm{SrCu}_{2} \mathrm{O}_{3}$ $[2,5]$, among others, reveal that even a small concentration of impurities reduces the spin gap. 
This feature has also been predicted by numerical calculations, such as the exact diagonalization and the Monte Carlo method for the Heisenberg ladder model [2, 6, 7].

Initially, most of the theoretical results concerning ladder systems were obtained from studies of the standard Heisenberg ladder. However, in contrast to its one-dimensional analogue, the Heisenberg ladder cannot be solved exactly. Subsequently, in order to understand the behaviour of spin ladder systems, other models with generalized interaction terms which guarantee integrability have been proposed [8-20]. Such generalized models qualitatively exhibit realistic physical properties such as the existence of a spin gap [8] and the prediction of magnetization plateaux at fractional values of the total magnetization [12]. However, although there is a considerable amount of work on integrable spin ladder systems in the literature, very few are concerned with the presence and influence of impurities. (One exception, however, is [18].)

The effect of impurities in spin chains and strongly correlated electron systems is well established to be significant, particularly in low-dimensional cases. When undertaking studies appealing to exact solutions afforded by integrable systems, it is possible to incorporate impurities into the system without violating integrability. This can be achieved via two methods. In the first case, the impurities are given by changing the representation of the underlying Yang-Baxter algebra structure at some lattice sites from the fundamental representation (i.e., that given by the $R$-matrix itself) to some other representation. In this context, several versions of both the Heisenberg and $t-J$ models have been investigated [21$23,25]$. Another possibility is to introduce the impurities by way of inhomogeneities in the transfer matrix of the system. This was explored, for instance, in [26-29].

Here we wish to adapt these two known methods used for one-dimensional systems to incorporate impurities into the quasi-one-dimensional case for the purpose of studying impurities in spin ladder systems. In particular, we will construct two integrable spin ladder models based on the $s u$ (4) algebra (Wang's model [8]) with impurities. These models have the property of having two coupling parameters, the rung coupling $J$ and the impurity strength $\Lambda$. The effect of these impurities on the phase transition between the gapped and gapless spin excitations of both models is investigated. We show that in one of the cases the gap does not depend explicitly on the impurity, while it does in the other case. Moreover, it turns out in the latter instance that the spin gap decreases by increasing the impurity strength $\Lambda$. In addition, if we consider a fixed value for $\Lambda$, it turns out that the gap decreases by increasing the concentration of impurities, in qualitative agreement with the experiments.

The paper is organized as follows. In section 2 we construct an integrable spin ladder system where the impurities are introduced by means of the dual representation of $s u(4)$. The model is solved exactly and the spin gap is determined. In section 3 we introduce another integrable spin ladder model, where the impurities are incorporated through the inhomogeneities in the transfer matrix. The Bethe ansatz equations, the energies, the spin gap and the phase transition of the model are discussed in detail. The effect of the impurities for different values of the rung coupling, the impurity strength and the concentration of impurities is discussed in section 3. A summary of the main results is presented in section 4 .

\section{Type I impurity spin ladder system}

We begin by introducing the first model whose Hamiltonian reads

$$
H=\sum_{i=1}^{N} h_{i, i+1}+\frac{J}{2} \sum_{i=1}^{N}\left(\vec{\sigma}_{i} \cdot \vec{\tau}_{i}-1\right)+\sum_{i \in I}^{N} h_{i, \bar{i}, i+1}^{\mathrm{imp}}-\frac{J}{2} \sum_{\bar{i}=1}^{l}\left(\vec{\sigma}_{\bar{i}} \cdot \vec{\tau}_{\bar{i}}-1\right)
$$


where

$$
h_{i, \bar{i}, i+1}^{\mathrm{imp}}=\frac{2}{\Lambda-8} h_{i, i+1} Q_{i, \bar{i}}-\frac{2}{\Lambda} Q_{i, \bar{i}} h_{i, i+1}
$$

with $h_{i, i+1}$ and $Q_{i, \bar{i}}$ given by

$$
\begin{aligned}
& h_{i, i+1}=\frac{1}{4}\left(1+\vec{\sigma}_{i} \cdot \vec{\sigma}_{i+1}\right)\left(1+\vec{\tau}_{i} \cdot \vec{\tau}_{i+1}\right) \\
& Q_{i, \bar{i}}=\frac{1}{4}\left[1+\left(\sigma_{i}^{x} \sigma_{\bar{i}}^{x}-\sigma_{i}^{y} \sigma_{\bar{i}}^{y}+\sigma_{i}^{z} \sigma_{\bar{i}}^{z}\right)\right]\left[1+\left(\tau_{i}^{x} \tau_{\bar{i}}^{x}-\tau_{i}^{y} \tau_{\bar{i}}^{y}+\tau_{i}^{z} \tau_{\bar{i}}^{z}\right)\right] .
\end{aligned}
$$

Above $\vec{\sigma}_{i}$ and $\vec{\tau}_{i}$ are Pauli matrices acting on the site $i$ (or the impurity site $\bar{i}$ ) of the upper and lower legs of the ladder, respectively, $J$ is the coupling constant for the interactions across the rungs and $\Lambda$ is an arbitrary parameter which measures the impurity strength. $I$ is simply an index set with $l$ elements in the range $1,2, \ldots, N$. For each $i \in I$ we couple an impurity into the ladder, situated between the sites $i$ and $i+1$. For clarity, we will denote this impurity site by $\bar{i}$. We make the assumption that if $i \in I$ then $i \pm 1 \notin I$ since in this instance extra terms are needed in the Hamiltonian for integrability. Throughout, $N$ is the number of rungs (equivalently, the length of the ladder), $l$ is the number of impurities, and periodic boundary conditions are imposed.

Note here that besides the usual Heisenberg interactions along the legs and rungs we also have Heisenberg-type interactions between the lattice site $i$ and the impurity site $\bar{i}$, as well as biquadratic interactions (also involving the impurity site $\bar{i}$ ), whose existence in the ladder compounds is supported by recent neutron experiments [32] (more details about the physical importance of this type of interaction can be found in [33]). The other two terms in the Hamiltonian are due to the presence of the impurities: specifically there are Heisenbergtype interactions between the lattice site $i$ and the impurity site $\bar{i}$. There are also three-site interactions involving the lattice sites $i, i+1$ as well as the impurity site $\bar{i}$ and impurity rung interactions. Finally, we mention that the Hamiltonian for the ladder model based on the fundamental representation of the $s u(4)$ algebra [8] can be recovered from equation (1) by taking the limit $\Lambda \rightarrow \infty$.

The energy eigenvalues of the Hamiltonian (1) are given by

$$
E=N-2 J(N-l)-4 \sum_{i=1}^{M_{1}}\left(\frac{1}{\lambda_{i}^{2}+1}-\frac{J}{2}\right)
$$

where $\lambda_{i}$ are solutions to the Bethe ansatz equations (BAE) below. The BAE arise from the exact solution of the model through the algebraic nested Bethe ansatz method. A significant point here is that in the Bethe ansatz approach for combinations of the fundamental and dual representations there is no unique pseudo-vacuum. Rather one is forced to work with a subspace of pseudo-vacua. This approach was developed in the works of Abad and Rios for an integrable $s u(3)$ spin chain combining different representations (the fundamental and its dual) $[30,31]$ and in the integrable impurity $t-J$ model constructed by combining the fundamental and dual representations of the superalgebra $g l(2 / 1)$ [23] (see also [24] for an alternative approach). Here we employ the same strategy for the case of $S U(4)$ and its dual. The procedure is basically the same, except for the fact that there is an extra level in the nesting. 
For the present case the BAE read

$$
\begin{aligned}
& \left(\frac{\lambda_{l}-\mathrm{i}}{\lambda_{l}+\mathrm{i}}\right)^{N}=\prod_{l \neq i}^{M_{1}} \frac{\lambda_{l}-\lambda_{i}-2 \mathrm{i}}{\lambda_{l}-\lambda_{i}+2 \mathrm{i}} \prod_{j=1}^{M_{2}} \frac{\lambda_{l}-\mu_{j}+\mathrm{i}}{\lambda_{l}-\mu_{j}-\mathrm{i}} \\
& \prod_{j \neq l}^{M_{2}} \frac{\mu_{l}-\mu_{j}-2 \mathrm{i}}{\mu_{l}-\mu_{j}+2 \mathrm{i}}=\prod_{i=1}^{M_{1}} \frac{\mu_{l}-\lambda_{l}-\mathrm{i}}{\mu_{l}-\lambda_{i}+\mathrm{i}} \prod_{k=1}^{M_{3}} \frac{\mu_{l}-v_{k}-\mathrm{i}}{\mu_{l}-v_{k}+\mathrm{i}} \\
& \left(\frac{v_{l}-\Lambda+\mathrm{i}}{v_{l}-\Lambda-\mathrm{i}}\right)^{l} \prod_{k \neq l}^{M_{3}} \cdot \frac{v_{l}-v_{k}-2 \mathrm{i}}{v_{l}-v_{k}+2 \mathrm{i}}=\prod_{j=1}^{M_{2}} \frac{v_{l}-\mu_{j}-\mathrm{i}}{v_{l}-\mu_{j}+\mathrm{i}}
\end{aligned}
$$

The presence of the impurity can be detected explicitly through the presence of the parameter $\Lambda$ in the first term of the last equation above.

When $J>2$, the ground state is given by a product of rung singlets and the energy is $E_{0}=N-2 J(N-l)$. This state belongs to the subspace of pseudo-vacuum states and corresponds to the case $M_{1}=M_{2}=M_{3}=0$ of the BAE (6). To describe an elementary excitation, we choose $M_{1}=1$ and $M_{2}=M_{3}=0$ in the BAE (6) which gives the minimal excited state energy, $E_{1}=N-2 J(N-l)+2 J-4$.

The energy gap can easily be calculated and is found to be

$$
\Delta=2(J-2) \text {. }
$$

By solving $\Delta=0$ for $J$ we find the critical value $J^{\mathrm{c}}=2$, indicating the critical point at which the quantum phase transition from the dimerized phase to the gapless phase occurs. Note that there is no effect of the impurity $\Lambda$ on the gap.

The integrability of this model can be shown by the fact that it can be mapped to the following Hamiltonian, which can be derived from two different $R$-matrices ( $R$ and $R^{*}$ below) obeying the Yang-Baxter algebra for $J=0$, while for $J \neq 0$ the rung interactions take the form of a chemical potential term:

$$
H=\sum_{i=1}^{N} h_{i, i+1}-2 J \sum_{i=1}^{N} X_{i}^{00}+\sum_{i \in I}^{l} h_{i, \bar{i}, i+1}^{\mathrm{imp}}+2 J \sum_{\bar{i}=1}^{l} X_{\bar{i}}^{00}
$$

where $h_{i, \bar{i}, i+1}^{\mathrm{imp}}$ is given by the same expression as in equation (2), but now with $h_{i, i+1}$ and $Q_{i, \bar{i}}$ given by

$$
\begin{aligned}
& h_{i, i+1}=\sum_{\alpha, \beta=0}^{3} X_{i}^{\alpha \beta} X_{i+1}^{\beta \alpha} \\
& Q_{i, \bar{i}}=\sum_{\alpha, \beta=0}^{3} X_{i}^{\alpha \beta} X_{\bar{i}}^{\alpha \beta} .
\end{aligned}
$$

In the above, $X_{i}^{\alpha \beta}=\left|\alpha_{i}\right\rangle\left\langle\beta_{i}\right|$ are the Hubbard operators with $\left|\alpha_{i}\right\rangle$ being the orthogonalized eigenstates of the local operators $\vec{\sigma}_{i} \cdot \vec{\tau}_{i}$. 
The two different $R$-matrices associated with $S U(4)$ and its dual ( $R$ and $R^{*}$, respectively) are

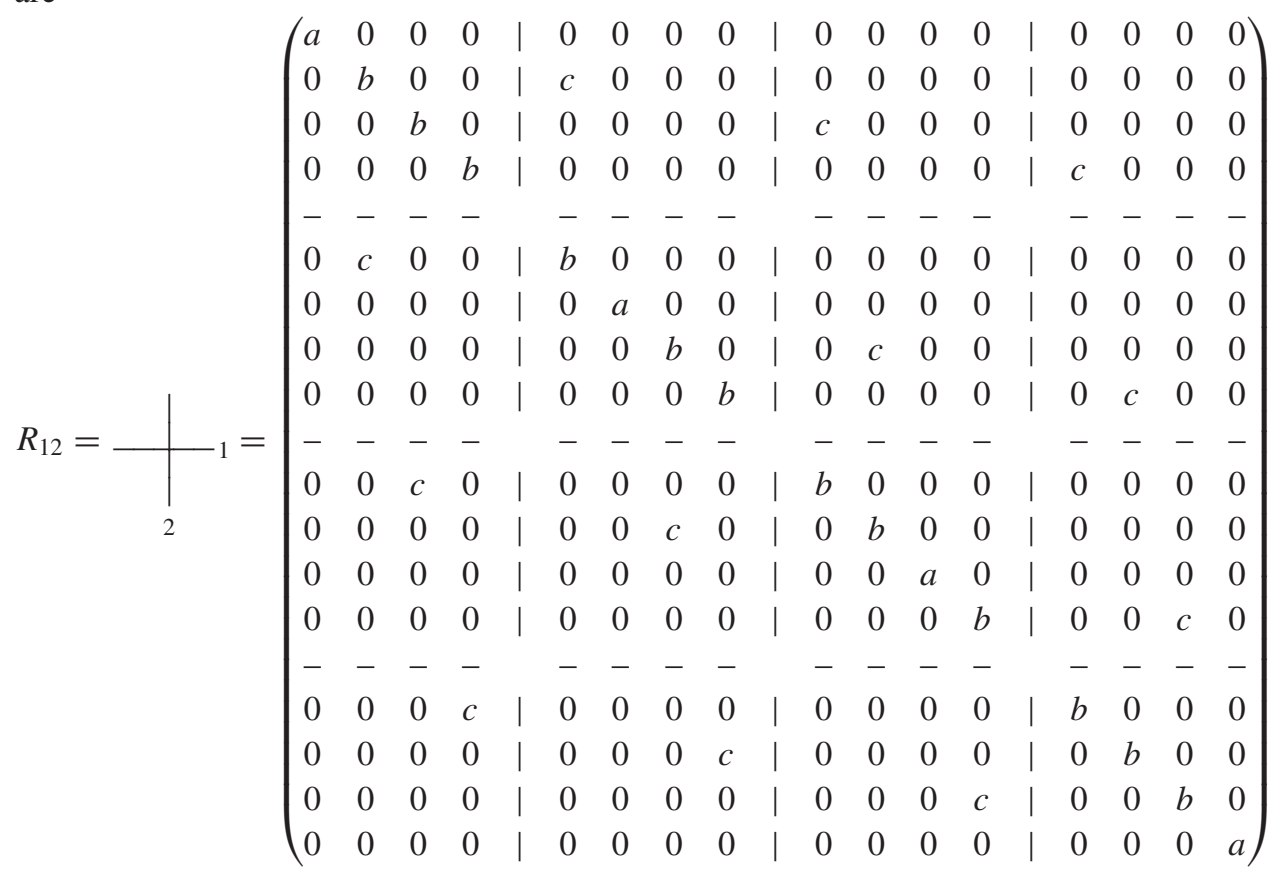

with

$$
\begin{aligned}
& a=-x / 2+1 \quad b=-x / 2 \quad c=1
\end{aligned}
$$

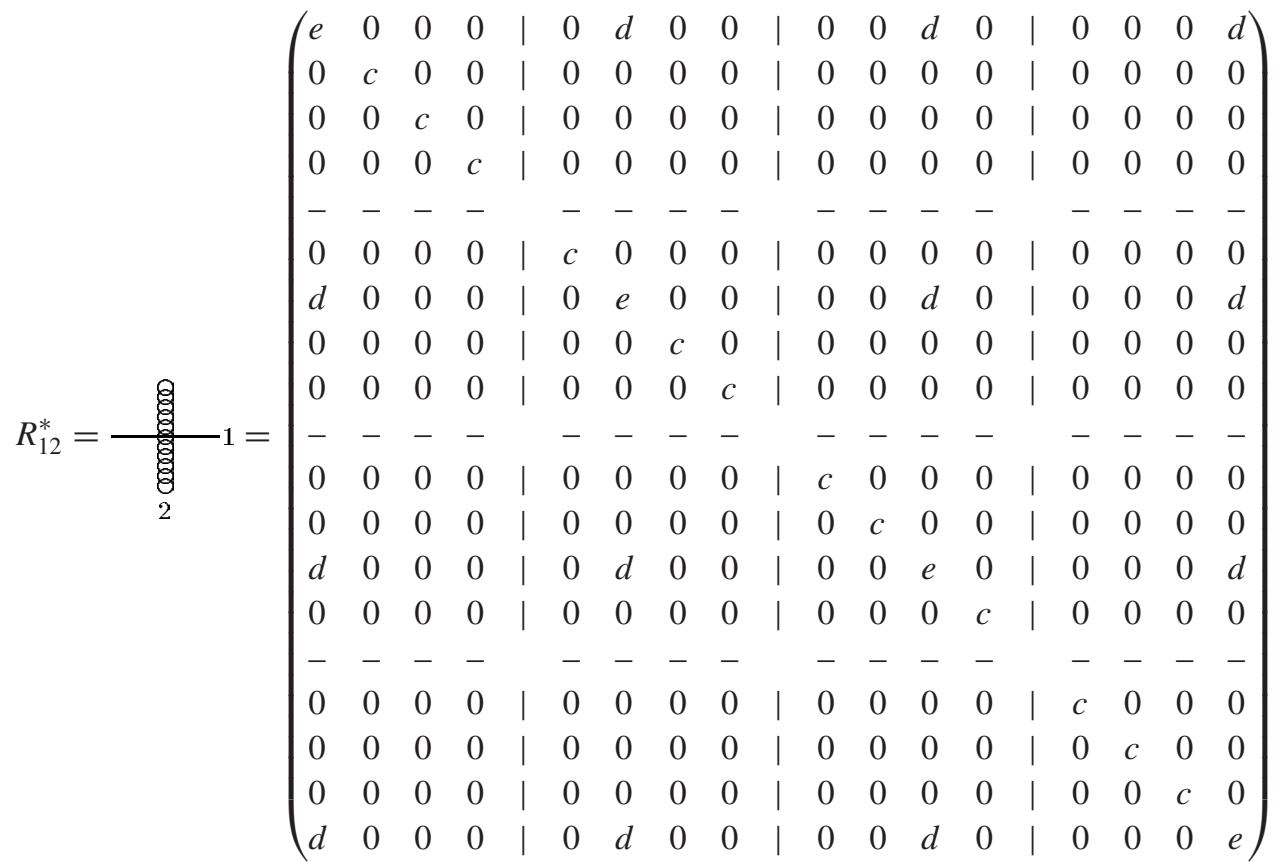


with

$$
c=1 \quad d=2 / x \quad e=1+\frac{2}{x} .
$$

The $R$-matrix (11) acts on the tensor product of the two four-dimensional spaces $V \otimes V$ while $R^{*}(12)$ acts on the tensor product of $V \otimes W$, where $W$ is the four-dimensional space for the dual representation. The matrices above satisfy the following Yang-Baxter equations:

$$
\begin{aligned}
& R_{12}(u-v) R_{13}(u) R_{23}(v)=R_{23}(v) R_{13}(u) R_{12}(u-v) \\
& R_{12}(u-v) R_{13}^{*}(u) R_{23}^{*}(v)=R_{23}^{*}(v) R_{13}^{*}(u) R_{12}(u-v)
\end{aligned}
$$

acting on $V \otimes V \otimes V$ and $V \otimes V \otimes W$, respectively.

Here we would like to mention that, in principle, any representation of $S U$ (4) can be chosen to introduce an impurity site into the chain. The choice of the dual representation is natural since it has the same dimension as the defining representation. This has the advantage that it allows us to identify the four states on which the dual representation acts with the rung states.

The impurity model is constructed with generic quantum spaces represented by $V$ and the impurity spaces $W$. To this end take some index set $I=\left\{p_{1}, p_{2}, \ldots, p_{l}\right\}, 1 \leqslant p_{i} \leqslant N$ and define

$$
X=\bigotimes_{i=1}^{N} X_{i}
$$

where

$$
\begin{array}{ll}
X_{i}=V & \text { if } \quad i \notin I \\
X_{i}=V \otimes W & \text { if } \quad i \in I .
\end{array}
$$

In other words for each $i \in I$ we are coupling an impurity into the lattice which will be situated between the sites $i$ and $i+1$.

Next we define the monodromy matrix

$$
T(u)=\bar{R}_{01}(u) \bar{R}_{02}(u) \ldots \bar{R}_{0 N}(u)
$$

where the subscript 0 denotes the auxiliary space and we have

$$
\begin{array}{ll}
\bar{R}_{0 i}(u)=R_{0 i}(u) & \text { for } \quad i \notin I \\
\bar{R}_{0 i}(u)=R_{0 i}(u) R_{0 \bar{i}}^{*}(u-\Lambda) & \text { for } \quad i \in I
\end{array}
$$

or in matrix form

$$
T(u)=\left(\begin{array}{cccc}
T_{1}^{1} & T_{2}^{1} & T_{3}^{1} & T_{4}^{1} \\
T_{1}^{2} & T_{2}^{2} & T_{3}^{2} & T_{4}^{2} \\
T_{1}^{3} & T_{2}^{3} & T_{3}^{3} & T_{4}^{3} \\
T_{1}^{4} & T_{2}^{4} & T_{3}^{4} & T_{4}^{4}
\end{array}\right)
$$

whose elements are operators acting on the quantum space $X$. A consequence of equations (13) and (14) is that $T(u)$ satisfies the Yang-Baxter algebra

$$
R_{12}(u-v) T_{13}(u) T_{23}(v)=T_{23}(v) T_{13}(u) R_{12}(u-v) .
$$


A picture of the monodromy matrix for the case $N=6$ and two impurities situated at impurity site $(\overline{2}$ and $\overline{5})$, is presented below

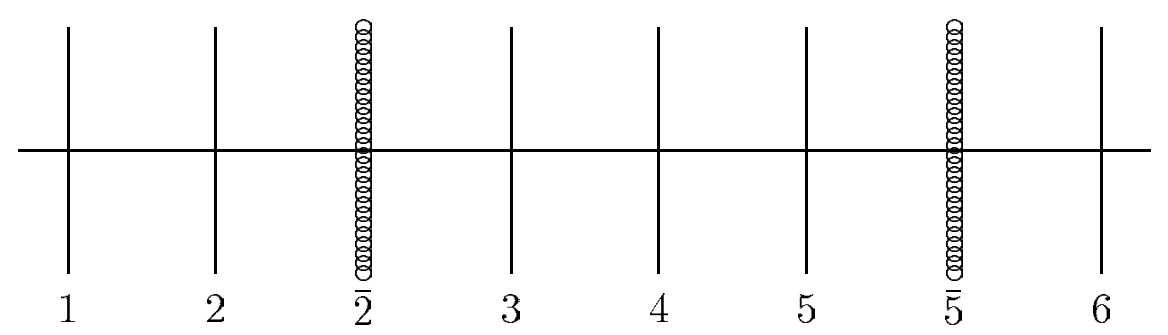

Note here that the impurities are incorporated into the system through the inclusion of the operator $R^{*}(u-\Lambda)$ in the transfer matrix.

The transfer matrix is defined by

$$
\tau(u)=\operatorname{tr}_{0} T(u)
$$

from which the Hamiltonian (8) (for $J=0$ ) is obtained through

$$
H=-\left.2 \frac{\partial}{\partial u} \ln \tau(u)\right|_{u=0}
$$

In this derivation we have used the properties

$$
\begin{aligned}
& {\left[R_{i, \bar{i}}^{*}(-u)\right]^{-1}=1+\frac{2}{u-8} Q_{i, \bar{i}}} \\
& Q_{i, \bar{i}} \cdot P_{i, i+1} \cdot Q_{i, \bar{i}}=Q_{i, \bar{i}} \\
& Q_{i, \bar{i}} \cdot Q_{i, \bar{i}}=4 Q_{i, \bar{i}} .
\end{aligned}
$$

This simplifies the calculations and is responsible for the structure of the Hamiltonian.

\section{Type II impurity spin ladder system}

Let us now introduce another spin ladder model with impurities, whose Hamiltonian reads

$$
H=\sum_{i=1}^{N} h_{i, i+1}+\frac{J}{2} \sum_{i=1}^{N}\left(\vec{\sigma}_{i} \cdot \vec{\tau}_{i}-1\right)+\sum_{i \in I}^{N} h_{i, \bar{i}, i+1}^{\mathrm{imp}}+\frac{J}{2} \sum_{\bar{i}=1}^{l}\left(\vec{\sigma}_{\bar{i}} \cdot \vec{\tau}_{\bar{i}}-1\right)
$$

where

$h_{i, i+1}=P_{i, i+1}=\frac{1}{4}\left(1+\vec{\sigma}_{i} \cdot \vec{\sigma}_{i+1}\right)\left(1+\vec{\tau}_{i} \cdot \vec{\tau}_{i+1}\right)$

$h_{i, \bar{i}, i+1}^{\mathrm{imp}}=\frac{4}{4-\Lambda^{2}}\left\{P_{i, \bar{i}}+P_{\bar{i}, i+1}-P_{i, i+1}\right\}+\frac{2 \Lambda}{4-\Lambda^{2}}\left\{P_{i, i+1} P_{i, \bar{i}}-P_{i, \bar{i}} P_{i, i+1}\right\}-\frac{2 \Lambda}{4-\Lambda^{2}} I_{i, \bar{i}}$

and periodic boundary conditions are imposed. Above, $N$ and $I$ have the same meanings as before. The total number of impurities is $l$, such that the concentration of impurities of the model $k$ can be defined as

$$
k=\frac{l}{N} \text {. }
$$

Assuming as before that if $i \in I$ then $i \pm 1 \notin I$, the imposition of not having neighbouring impurities implies that $k$ cannot exceed $1 / 3$. From the physical point of view this restriction does not cause any difficulty, since most of the experiments are carried out in the underdoped 
regime [5]. The Hamiltonian (24) contains two coupling parameters, the rung coupling $J$ and the impurity strength $\Lambda$. Essentially, the competition between these two terms will determine the physical properties of the model. We also note that Wang's ladder system [8] with length $N+l$ can be recovered by taking the limit $\Lambda=0$.

The energy eigenvalues of the Hamiltonian (24) are given by

$$
E=(1-2 J) N+\left(\frac{2}{2+\Lambda}-2 J\right) l-4 \sum_{i=1}^{M_{1}}\left(\frac{1}{\lambda_{i}^{2}+1}-\frac{J}{2}\right)
$$

where $\lambda_{i}$ are solutions to the Bethe ansatz equations obtained through the algebraic nested Bethe ansatz with three levels. In contrast to the previous case, the derivation here is straightforward due to the existence of a unique pseudo-vacuum. Since this approach is well known in the literature [34], we only present the final result

$$
\begin{aligned}
& \left(\frac{\lambda_{l}-\mathrm{i}}{\lambda_{l}+\mathrm{i}}\right)^{N}\left(\frac{\lambda_{l}-\mathrm{i}(1-\Lambda)}{\lambda_{l}+\mathrm{i}(1+\Lambda)}\right)^{l}=\prod_{l \neq i}^{M_{1}} \frac{\lambda_{l}-\lambda_{i}-2 \mathrm{i}}{\lambda_{l}-\lambda_{i}+2 \mathrm{i}} \prod_{j=1}^{M_{2}} \frac{\lambda_{l}-\mu_{j}+\mathrm{i}}{\lambda_{l}-\mu_{j}-\mathrm{i}} \\
& \prod_{j \neq l}^{M_{2}} \frac{\mu_{l}-\mu_{j}-2 \mathrm{i}}{\mu_{l}-\mu_{j}+2 \mathrm{i}}=\prod_{i=1}^{M_{1}} \frac{\mu_{l}-\lambda_{i}-\mathrm{i}}{\mu_{l}-\lambda_{i}+\mathrm{i}} \prod_{k=1}^{M_{3}} \frac{\mu_{l}-v_{k}-\mathrm{i}}{\mu_{l}-v_{k}+\mathrm{i}} \\
& \prod_{k \neq l}^{M_{3}} \frac{v_{l}-v_{k}-2 \mathrm{i}}{v_{l}-v_{k}+2 \mathrm{i}}=\prod_{j=1}^{M_{2}} \frac{v_{l}-\mu_{j}-\mathrm{i}}{v_{l}-\mu_{j}+\mathrm{i}} .
\end{aligned}
$$

For $J>\frac{2}{\lambda^{2}+1}$ the ground state is given by the product of rung singlets and the energy is $E_{0}=\left(\frac{2}{2+\Lambda}-2 J\right) l+(1-2 J) N$, corresponding as before to the case $M_{1}=M_{2}=M_{3}=0$ of the BAE (28). To describe an elementary excitation, we choose $M_{1}=1$ and $M_{2}=M_{3}=0$ in the BAE (28), which gives the minimal excited state energy. This solution depends on the concentration of impurities $k=l / N$. Let us consider first the case $k=1 / 3$, which leads to the following imaginary solution for the variable $\lambda$ :

$$
\lambda=-\frac{\mathrm{i}}{12}\left(-3 \Lambda+\frac{3^{2 / 3}\left(-16+3 \Lambda^{2}\right)}{K}+3^{1 / 3} K\right)
$$

with $K$ given by

$$
K=\left(-9 \Lambda^{3}+4 \sqrt{3} \sqrt{256-144 \Lambda^{2}+27 \Lambda^{4}}\right)^{1 / 3} .
$$

Note that the solution of the BAE $\lambda$ above depends explicitly on the impurity $\Lambda$. This relation is illustrated in figure 1.

The energy gap can be calculated using the Bethe ansatz solution and has the form

$$
\Delta=2\left(J-\frac{2}{\lambda^{2}+1}\right) \text {. }
$$

Here, in contrast to the previous case, the impurity does affect the gap. By solving $\Delta=0$ for $J$ we find the critical value $J^{\mathrm{c}}=\frac{2}{\lambda^{2}+1}$, indicating the critical line at which the quantum phase transition from the dimerized phase to the gapless phase occurs. The phase transition line is shown in figure 2. We would like to mention that in the context of spin chains, the effect of impurities in the low energy spectrum and in the study of the phase transitions was investigated recently in [35].

Note that by increasing the impurity strength $\Lambda$, the critical value $J^{\mathrm{c}}$ also increases. A further analysis of both graphs together with the gap expression (30) reveals that there is a reduction in the gap by increasing the impurity strength $\Lambda$. This result can be easily confirmed by an inspection of figure 3 for different values of $J$. 


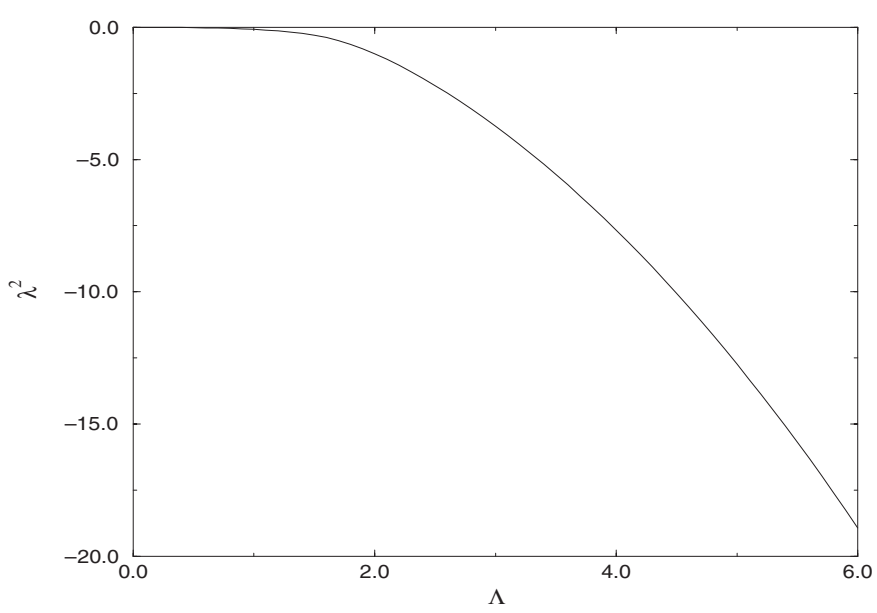

Figure 1. This graph shows how the solution of the $\operatorname{BAE}\left(\lambda^{2}\right)$ depends on the impurity $\Lambda$.

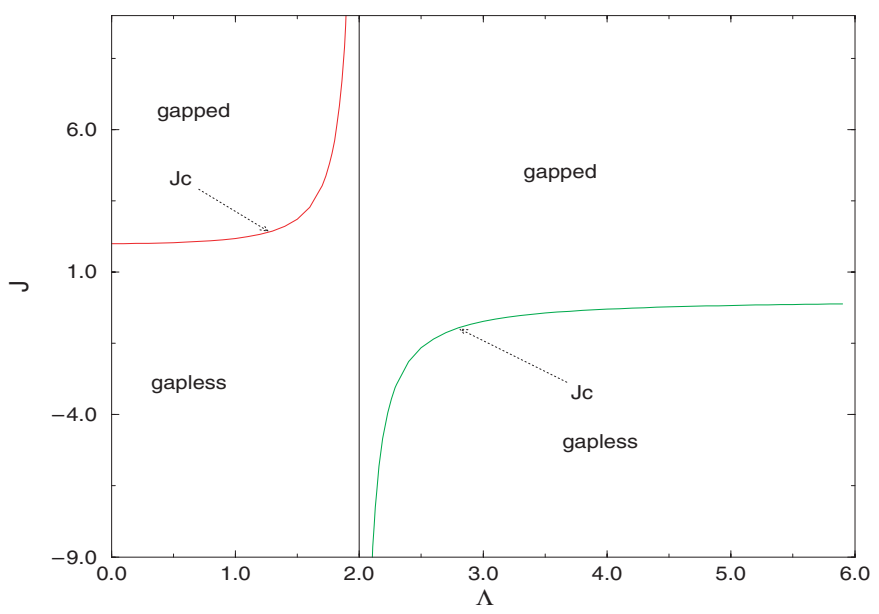

Figure 2. Rung coupling $J$ versus impurity $\Lambda$. This graph represents the phase diagram. The curve $\left(J^{\mathrm{c}}=2 /\left(\lambda^{2}+1\right)\right)$ divides the gapped and gapless phases.

A similar analysis can be performed for other impurity concentrations $k$, although in general just a numerical solution of the BAE (28) is possible. We have observed the same qualitative behaviour as in the case $k=1 / 3$. Therefore, we will just present the most significant results for further discussion. Figure 4, for example, illustrates the behaviour of the solution of the BAE associated with the minimal excited state as a function of the impurity strength for different concentrations. The fact that the gap decreases by increasing the impurity strength $\Lambda$ for a fixed rung coupling $J$ is also observed for other concentrations, as illustrated in figure 5. Another interesting property that can be easily confirmed by an inspection of this figure is the decreasing of the gap by increasing the concentration of impurities $k$ for a fixed $J$ and $\Lambda$, in qualitative agreement with the experiments [2].

The exact solvability of this model, as for the previous case, can be shown by the fact that it can be mapped to an Hamiltonian with the same form as equation (8), where $h_{i, \bar{i}, i+1}^{\mathrm{imp}}$ is 


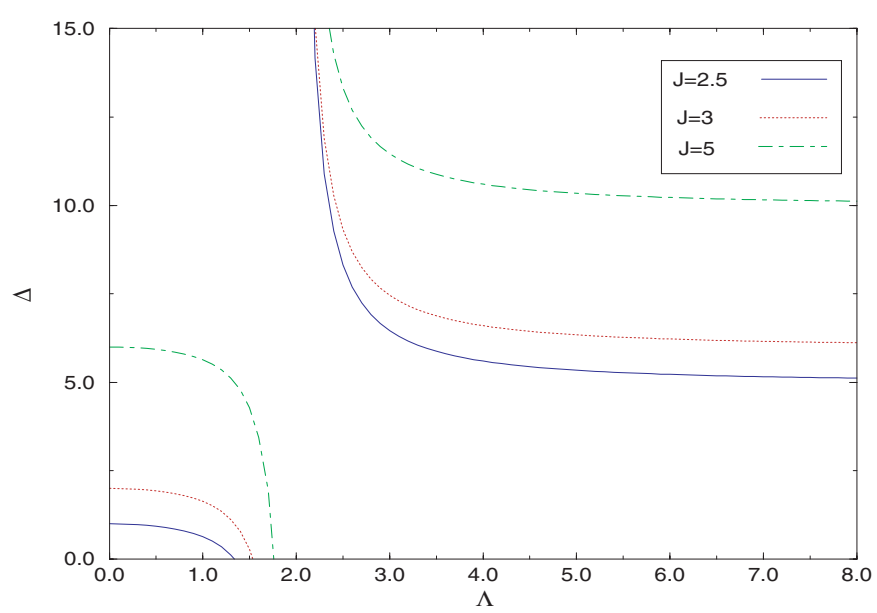

Figure 3. This graph shows how the spin gap $\Delta$ depends on the impurity strength $\Lambda$ for different values of the rung coupling $J$.

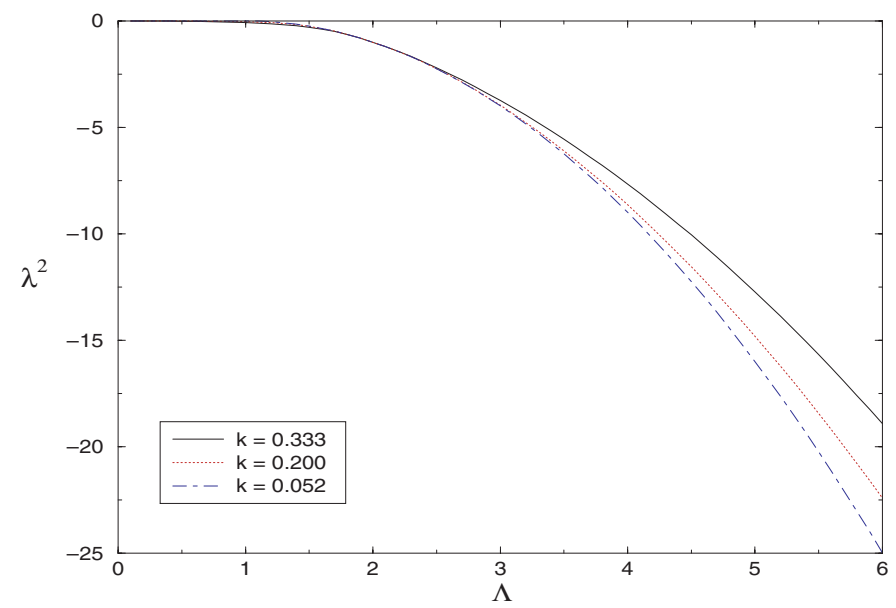

Figure 4. This figure indicates how the solution of the BAE $\lambda^{2}$ depends on the impurity strength $\Lambda$ for different concentrations $k$ for the impurities.

given by equation (26) with $h_{i, i+1}$ written in terms of the Hubbard operators as in equation (9). The difference in the present case is the definition of the monodromy matrix, given by

$$
T(u, \Lambda)=\bar{R}_{01}(u) \bar{R}_{02}(u) \ldots \bar{R}_{0 N}(u)
$$

where we have

$$
\begin{aligned}
& \bar{R}_{0 i}(u)=R_{0 i}(u) \quad \text { for } \quad i \notin I \\
& \bar{R}_{0 i}(u)=R_{0 i}(u) R_{0 \bar{i}}(u-\Lambda) \quad \text { for } \quad i \in I
\end{aligned}
$$

and the $R$-matrix (equation (11)) obeys the Yang-Baxter equation (13). Note from the above equation that here the impurity $\Lambda$ is associated with the same $R$-matrix, in contrast to the previous case (see equation (16)), where it was associated with a different one $\left(R^{*}\right)$. This is the main reason for the difference in the structure of the Hamiltonians. Next the transfer matrix is defined as in equation (19) from which the Hamiltonian is obtained through the use 


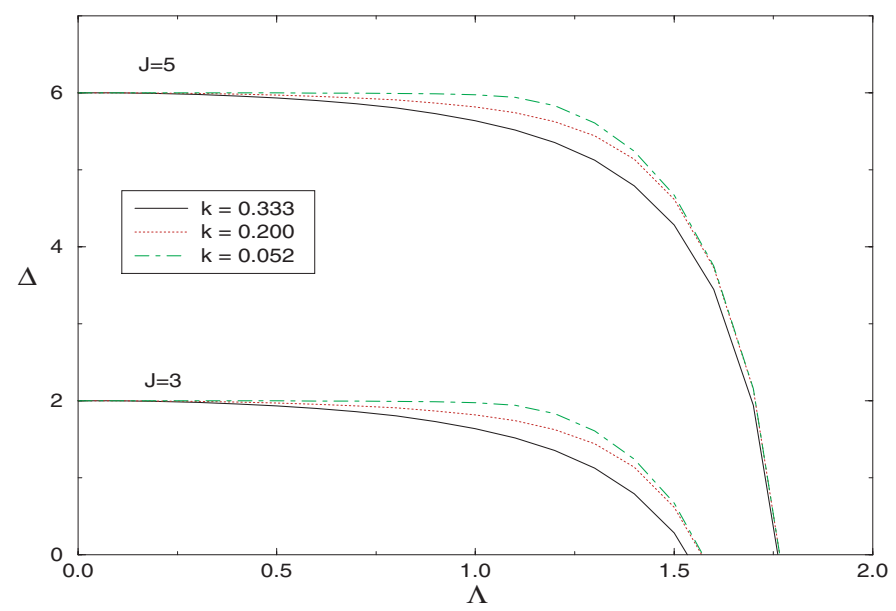

Figure 5. This graph shows how the spin gap $\Delta$ depends on the impurity strength $\Lambda$ for different concentrations and for different values of the rung coupling $J$. Note that $\Delta$ decreases by increasing the concentration for any value of $J$ and $\Lambda$.

of equation (20). The proof follows analogously to the previous type I impurity ladder, except for the fact that the properties (21), (22) and (23) are obviously not used.

Finally, we would like to mention that in both cases a different parametrization at each impurity site could be used. Although this does not considerably change the structure of the Hamiltonians and the Bethe ansatz equations (see, for example, [23] for an example of this general parametrization for the case of the $t-J$ model with impurities), the existence of different parameters would make the numerical analysis more complex.

\section{Summary}

To summarize, we have introduced two integrable spin ladder models with impurities characterized by two coupling parameters, the rung coupling $J$ and the impurity strength $\Lambda$. In a particular limit both models reduce to that introduced by Wang [8] based on the $s u(4)$ Lie algebra. The Bethe ansatz equations as well as the energy expressions for the models were presented. We have shown that in one of the cases the impurities do not affect the gap of the system, while in the other case there is a non-trival dependence. In fact, it is found that the gap decreases by increasing the impurity strength $\Lambda$. Moreover for a fixed $\Lambda$, a reduction in the spin gap by increasing the impurity concentration is also observed, in qualitative agreement with the experiments.

\section{Acknowledgments}

APT thanks CNPq-Conselho Nacional de Desenvolvimento Científico e Tecnológico for financial support. AF thanks DAAD (Deutscher Akademischer Austauschdienst) and FAPERGS (Fundação de Amparo a Pesquisa do Estado do Rio Grande do Sul) for financial support. XWG thanks FAPERGS for financial support. JL thanks the ARC (Australian Research Council) for financial support. AF thanks the Institut für Theoretische Physik der Freie Universität Berlin for their kind hospitality. 


\section{References}

[1] Dagotto E and Rice T M 1996 Science 271618

[2] Dagotto E 1999 Rep. Prog. Phys. 621525

[3] Azuma M, Hiroi Z and Takano M 1994 Phys. Rev. Lett. 733463

[4] Ishida K, Kitaoka Y, Tokunaga S, Matsumoto S and Asayama K 1996 Phys. Rev. B 532827

[5] Hewitt $\mathrm{K} \mathrm{C}$ and Irwin J C 2000 Doping dependence of the superconducting gap in $\mathrm{Bi}_{2} \mathrm{Cr}_{2} \mathrm{CaCu}_{2} \mathrm{O}_{8}+\delta$, Preprint cond-mat/0012413

[6] Iino Y and Imada M 1996 J. Phys. Soc. Japan 653728

[7] Motone Y, Katoh N, Furukama N and Imada M 1996 J. Phys. Soc. Japan 651949

[8] Wang Y 1999 Phys. Rev. B 609236

[9] Albeverio S, Fei S-M and Wang Y 1999 Europhys. Lett. 47364

[10] Batchelor M T and Maslen M 1999 J. Phys. A: Math. Gen. 32 L377

[11] Frahm H and Kundu A 1999 J. Phys. C: Solid State Phys. 11 L557

[12] de Gier J and Batchelor M T 2000 Phys. Rev. B 62 R3584

[13] Batchelor M T and Maslen M 2000 J. Phys. A: Math. Gen. 33443

[14] Batchelor M T, de Gier J, Links J and Maslen M 2000 J. Phys. A: Math. Gen. 33 L97

[15] de Gier J, Batchelor M T and Maslen M 2000 Phys. Rev. B 6115196

[16] Links J and Foerster A 2000 Phys. Rev. B 6265

[17] Kundu A 2000 J. Math. Phys. 41721

[18] Wang Y and Schlottmann P 2000 Phys. Rev. B 623845

[19] Foerster A, Hibberd K E, Links J R and Roditi I 2001 J. Phys. A: Math. Gen. 34 L25

[20] Anbjorn J, Arnaudon D, Sedrakyan A, Sedrakyan T and Sorba P 2001 J. Phys. A: Math. Gen. 345887

[21] Bedürftig G, Essler F H L and Frahm H 1996 Phys. Rev. Lett. 775098

Bedürftig G, Essler F H L and Frahm H 1997 Nucl. Phys. B 498697

[22] Foerster A, Links J and Tonel A P 1999 Nucl. Phys. B 552707

[23] Links J and Foerster A 1999 J. Phys. A: Math. Gen. 32147

[24] Martins M 1999 Integrable mixed vertex models from braid-monoid algebra Preprint solv-int/9903006

[25] Andrei N and Johannesson H 1984 Phys. Lett. A 100108

[26] Schmitteckert P, Schwab P and Eckern U 1995 Europhys. Lett. 30543

[27] Eckle H-P, Punnoose A and Römer R 1997 Europhys. Lett. 39293

[28] Guan X-W, Grimm U, Römer R A and Scheiber M 2000 J. Phys. A: Math. Gen. 333863

[29] Bares P-A 1994 Exact results for a one dimensional $t-J$ model with impurities Preprint cond-mat/9412011

[30] Abad J and Ríos M 1996 Phys. Rev. B 5314000

[31] Abad J and Ríos M 1997 J. Phys. A: Math. Gen. 305887

[32] Matsuda M et al 2000 Phys. Rev. B 628903

[33] Neresyan A A and Tsvelick A T 1997 Phys. Rev. Lett. 783939 Kolezhuk A K and Mikeska H-J 1998 Phys. Rev. Lett. 802709 Wang Y, Essler F, Fabrizio M and Neresesyan A 2001 Preprint cond-mat/0112249

[34] Babelon O, de Vega H J and Viallet C M 1982 Nucl. Phys. B 200266 Kulish P P and Reshetikhin N Yu 1981 Sov. Phys.-JETP 53108 Kulish P P and Reshetikhin N Yu 1983 J. Phys. A: Math. Gen. 16 L591 de Vega H J and Lopes E 1991 Phys. Rev. Lett. 67489 Schultz C L 1983 Physica A 12271

[35] Contucci P, Nachtergaele B and Spitzer W 2002 Phys. Rev. B 66064429 